\title{
Biomimetic Bilayered Gelatin-Chondroitin 6 Sulfate-Hyaluronic Acid Biopolymer as a Scaffold for Skin Equivalent Tissue Engineering
}

\author{
*Tzu-Wei Wang, *Hsi-Chin Wu, *Yi-Chau Huang, † $\$$ Jui-Sheng Sun, and *§Feng-Huei Lin \\ *Institute of Biomedical Engineering, College of Medicine and College of Engineering, National Taiwan University; †Institute \\ of Rehabilitation Science and Technology, National Yang-Ming University; $\neq$ Department of Orthopedic Surgery, Taipei \\ Municipal Yang-Ming Hospital; and \$Department of Biomedical Engineering, National Taiwan University Hospital,
} Taipei, Taiwan

\begin{abstract}
In order to develop an adequate scaffold for skin tissue engineering, a bilayered gelatin-chondroitin 6 sulfate-hyaluronic acid membrane with a different pore size on either side was prepared. A rete ridges-like topographic microporous structure, which provided the paracrine crosstalk in the epithelial-mesenchymal interactions, was formed. Chondroitin-6-sulfate and hyaluronic acid were incorporated within the gelatin membrane to mimic skin composition and create an appropriate microenvironment for cell proliferation, differentiation, and migration. In the study, the lower layer of the membrane (pore size: $150 \mu \mathrm{m})$ was seeded with dermal fibroblasts and acted as the feeder layer for keratinocyte inoculation. Meanwhile, the upper layer (pore size: $20-50 \mu \mathrm{m}$ ) was seeded with keratinocytes for epidermalization. The dermal fibroblasts were dynamically seeded in a self-designed spinner flask for more even cell distribution. The keratinocytes were
\end{abstract}

cultured in submerged conditions for 5 days and then in an air-liquid interface condition for further differentiation. After being cultured for 21 days, the upper layer, seeded with keratinocytes, developed into an epidermislike structure while the lower part, which was seeded with dermal fibroblasts developed into a dermis-like structure. A histological examination and immunostain were used to prove that keratinocytes maintain their phenotype and stratified epidermis layers were formed within 21 days. In brief, the bilayered skin substitute with biological dermal analog and epidermal structure was successfully fabricated. From this study, we can suggest that the culture model is suitable for autologous skin equivalent preparation. Key Words: Dermal fibroblasts-Keratinocytes-Bilayered membrane-Skin equivalent-Tissue engineering.
Theoretically, an autogenous full-thickness skin graft is the best grafting choice for burn patients. However, those with extensive burns who have lost most of their dermis and epidermis are always in shortage of supply for harvesting full-thickness skin for grafting. Although full-thickness autografts effectively induce skin regeneration on the wound site, it can also create a new wound with uncosmetic hypertrophic scarring and pigmentation on the donor site. In clinical medicine, allograft and/or commercialized artificial skin have been used in extensive burn patients to promote dermis recovery, to evade bacte-

Received March 2005; revised October 2005.

Address correspondence and reprint requests to Dr. Feng-Huei Lin, Institute of Biomedical Engineering, National Taiwan University, No. 1, Sec. 1, Jen-Ai Road, Taipei, Taiwan. E-mail: double@ ha.mc.ntu.edu.tw rial contamination, and to prevent body fluid and body temperature loss. After the regeneration of the dermis, the cadaver or commercialized artificial skin (1-3) will be removed, and then a split-thickness autograft will be applied on the wound bed for epidermis growth $(4,5)$. This so-called "two-step grafting" process is now the orthodox procedure in clinical practice for extensive burn patients. Nevertheless, the extensive burn patients are still limited by the availability of donor sites for split-thickness autografts.

Two decades ago, Rheiwald and Green started the research on in vitro large-scale keratinocyte culture (6-8). Later, the keratinocyte sheet was successfully prepared in vitro and then applied on the burn area for epidermis growth. This method was a tremendous step forward in the treatment of large burns, but the cultured epidermal sheets still could not provide satisfactory skin coverage due to subsequent wound 
contraction $(9,10)$. Later, researchers prolonged the in vitro culture time of keratinocytes, but the high percentage of differentiated keratinocytes lost their continuous proliferative capacities in the epidermal renewal process. Furthermore, the complicated "twostep grafting" is both inconvenient for the patients and labor-intensive in the clinical practice. The concept of skin equivalent preparation for the treatment of extensive burn patient remains an unsolved problem (11-13).

In the study, a gelatin-chondroitin-6-sulfatehyaluronic acid (gelatin-C6S-HA) biopolymer was developed to mimic the skin extracellular matrix as the scaffold for skin tissue engineering. Gelatin-C6SHA could provide an optimum environment for cell growth, and then organized into a real skin tissue. In the study, a bilayered gelatin-C6S-HA membrane with different pore sizes on either side was prepared as the scaffold for skin equivalent tissue. The cell seeding was performed in a self-designed bioreactor to reach a better seeding condition. We suggest that this bilayered gelatin-C6S-HA membrane has potential in clinical applications for patients with extensive burns.

\section{MATERIALS AND METHODS}

\section{Preparation of porous bilayered gelatin-C6S-HA membrane}

Gelatin, chondroitin-6-sulfate (C6S)(C-4384), hyaluronic acid (HA) (H-5388), and 1-ethyl-3(3dimethyl aminopropryl) carbodiimide (E-1769) were all purchased from Sigma Chemical Co. (St. Louis, MO, U.S.A.). Gelatin (5 wt \%, 300 Bloom, from porcine skin) (G-2500 Sigma Co.) was dissolved in distilled water at $25^{\circ} \mathrm{C}$. Adequate amounts of C6S and $\mathrm{HA}$ were added to the gelatin solution until it became a slurry, with the final concentration at $0.05 \mathrm{wt} \%$ of $\mathrm{C} 6 \mathrm{~S}$ and $0.2 \mathrm{wt} \%$ of HA. The slurry solution was then well mixed at $30^{\circ} \mathrm{C}$ by magnetic stirrer for $1 \mathrm{~h}$. Next, $0.5 \mathrm{~mL}$ of the well-mixed slurry was poured into a preformed polyethylene mold $3 \mathrm{~cm}$ in diameter. For the preparation of the smallerpore $(20-50 \mu \mathrm{m})$-size upper layer, the formed slurry sheet was frozen at $-196^{\circ} \mathrm{C}$ for $30 \mathrm{~s}$. To prepare the larger-pore $(150 \mu \mathrm{m})$-size layer, another $1 \mathrm{~mL}$ of slurry solution was added onto the top of the frozen membrane and then left at room temperature for $3 \mathrm{~min}$. The membrane was then frozen at $-20^{\circ} \mathrm{C}$ for $3 \mathrm{~h}$ followed by lyophilizing at $-70^{\circ} \mathrm{C}$ overnight. The prepared bilayered membrane was cross-linked by $0.5 \mathrm{wt} \%$ 1-ethyl-3(3-dimethylaminopropyl) carbodiimide (EDC) solution overnight at $4^{\circ} \mathrm{C}$, sonicated five times in distilled water for $3 \mathrm{~min}$ to remove resid- ual EDC, frozen at $-20^{\circ} \mathrm{C}$ for $2 \mathrm{~h}$, and then lyophilized at $-70^{\circ} \mathrm{C}$. The cross-linked steps were repeated twice to stabilize the membrane without quick degradation during the culture period.

\section{Culture of keratinocytes and dermal fibroblasts}

Fresh adult human foreskin biopsies were washed using phosphate buffered saline with $1 \%$ penicillinstreptomycin-gentamycin antibiotic (Gibco Invitrogen Co., Burlington, Ontario, Canada). Specimens were then chopped into small fragments. The fragments were immersed in $40 \mathrm{U} / \mathrm{mg}$ thermolysin (P-1512, Sigma Co.) at $4^{\circ} \mathrm{C}$ overnight. To isolate the keratinocytes, the epidermis was separated from the dermis with forceps and then incubated in $0.1 \%$ ethylenediaminetetraacetic acid (EDTA) solution with $0.05 \%$ trypsin (Cat no. $15400-054$, Gibco Invitrogen Co.) for $15 \mathrm{~min}$ at $37^{\circ} \mathrm{C}$. The isolated keratinocytes were cultivated and expanded by commercially available serum-free/chemically defined culture MCDB 153 medium with indicated medium supplement (E-0151, EpiLife, Sigma Co.) in a 9-cm Petri dish. The dermis was treated with $0.2 \%$ collagenase (C-0130, Sigma Co.) at $37^{\circ} \mathrm{C}$ for $1 \mathrm{~h}$ to harvest the fibroblasts. The harvested dermal fibroblasts were cultured in Dulbecco's Modified Eagle's Medium (SH30022.02, HyClone, Logan, UT, U.S.A.) supplemented with 10\% FBS (Cat no. 12003-500M, JRH Bioscience, Lenexa, KS, U.S.A.) at $37^{\circ} \mathrm{C}, 5 \% \mathrm{CO}_{2}$.

\section{Skin equivalent preparation}

Dermal fibroblasts were inoculated in the lower part (with larger pore size) of the gelatin-C6S-HA membrane for 3 days to form the dermis equivalent. The membrane was taken out and the keratinocytes were seeded on the upper layer for epidermis-equivalent formation. The dermal fibroblasts were dynamically seeded in a self-designed $125-\mathrm{mL}$ spinner flask with $80 \mathrm{~mL}$ medium and cell density of $2 \times 10^{4}$ cells/ $\mathrm{mL}$ (Fig. 1a,b). The membrane was placed in a standing-still tissue cassette. A $2.5-\mathrm{cm}$ magnetic stir bar was spun at a rate of $80 \mathrm{rpm}$. The flask was designed in such a way that the dermal fibroblasts could only be caught in one side (larger pore size) of the membrane with a homogeneous cell distribution. The $1 \mathrm{~mL}$ well-mixed keratinocyte cell suspension containing culture medium $\left(2 \times 10^{5}\right.$ cells $\left./ \mathrm{mL}\right)$ was sprayed directly onto the surface of the gelatin-C6SHA scaffold for epidermis development. The keratinocyte-seeded membrane layer was submerged for 5 days in keratinocyte serum-free medium for proliferation and basal lamina formation. Then, the keratinocyte-seeded membrane was shifted to transwell and cultured in air-liquid interface conditions 
(a)

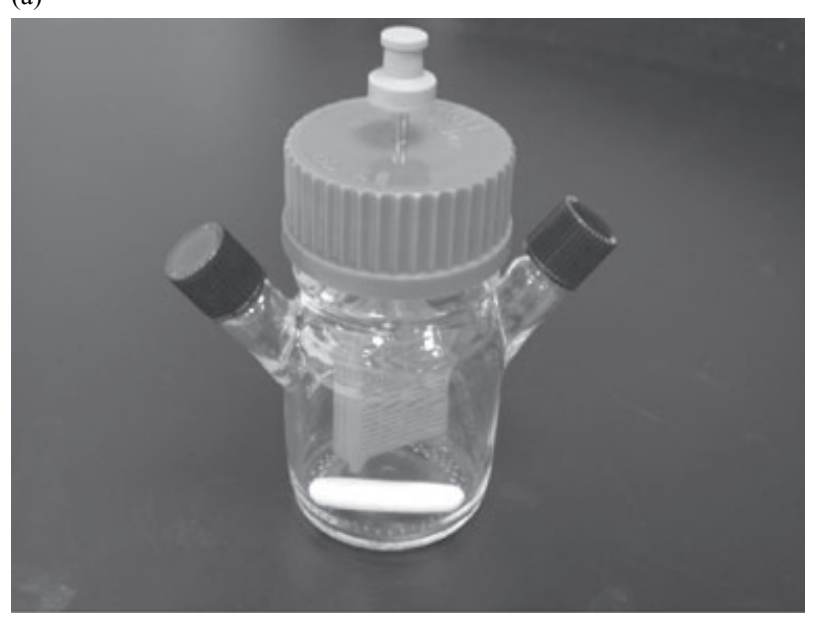

(b)

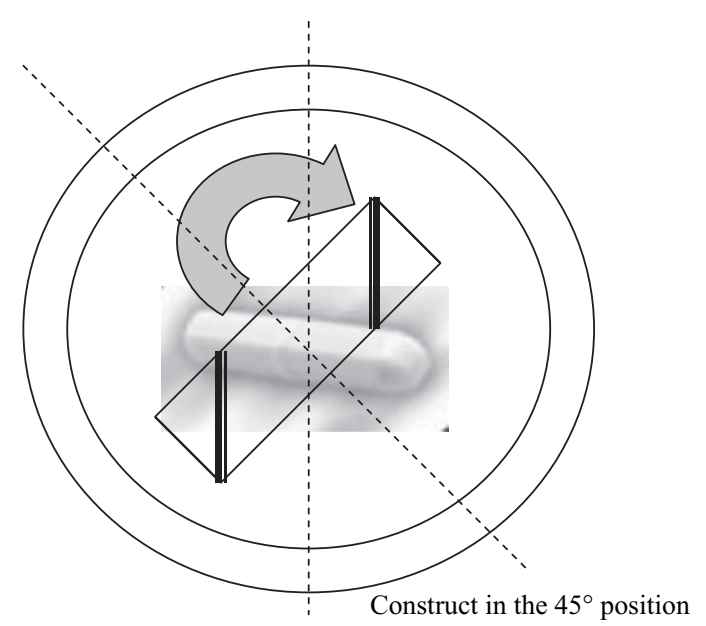

FIG. 1. Self-designed spinner flask bioreactor. This is designed to enable dermal fibroblasts seeding and growth. (a) Dynamic seeding method with spinner flask. (b) Top view of the bioreactor: the construct is at a $45^{\circ}$ position for maximum dermal fibroblasts adhesion density.

for 21 days to facilitate the epidermalization process $(14,15)$.

\section{Colorimetric MTT (Tetrazolium) assay for cell viability (16)}

For the assay, dermal fibroblasts were incubated in 96-well plates in the presence of various scaffold culture conditions (Petri dish, gelatin, gelatin-C6S-HA). The cell density was $5 \times 10^{3}$ cells/well. After various time intervals $(1,3,5$, and 7 days), the supernatant was removed and $100 \mu \mathrm{L}$ of MTT solution (3-[4, 5-dimethylthialzolyl-2]-2,5-diphenyltetrazolium bromide) (MTT, Sigma) was added to each well. The plate was incubated at $37^{\circ} \mathrm{C}$ for $4 \mathrm{~h}$ to allow the formation of formazan crystal. The dark blue crystals were dissolved by acid-isopropanol and were aspi- rate to another new 96-well plate. Read the optic density on MicroElisa reader (Emax Science Corp., CA, U.S.A.)

Total DNA analysis for quantification of cell growth The Wizard Genomic DNA Purification Kit (Promega Co., Madison, WI, U.S.A.) was chosen for DNA extraction. The first step was to lyse the cells and the nuclei with nuclei lysis solution and then followed by an exhaustive RNase digestion step. The cellular proteins were then removed by saltprecipitation method so that the high molecular weight genomic DNA is left in the solution. Finally, the genomic DNA is concentrated and desalted by isopropanol precipitation. The ratio of $260 \mathrm{~nm}$ and $280 \mathrm{~nm}$ absorption band on UV-Vis spectrum should be in the range of 1.8-2.0 to yield a good deproteinization.

\section{Scanning electron microscope (SEM) for cell morphology}

For the SEM examination, the gelatin-C6S-HA skin equivalents were fixed in $4 \%$ paraformaldehyde in $0.1 \mathrm{M}$ phosphate buffer solution for $2 \mathrm{~h}$ and then dried with a series of increasing graded alcohols followed by critical-point drier processing. The surfaces of the membrane or skin equivalent were coated with an ultrathin layer of gold/Pt in an ion sputter physical vapor deposition (PVD) chamber. The average diameter of pore sizes was analyzed with arbitrary matrix zone.

\section{Histology and immunohistochemistry}

For the light microscopic examination, the specimens were fixed in $10 \%$ formalin at $25^{\circ} \mathrm{C}$ for $2 \mathrm{~h}$, dried with graded alcohols, and then embedded in paraffin. A serial of 3-6 $\mu \mathrm{m}$ sections were taken across or parallel to the specimen, mounted on slides and then stained with hematoxylin and eosin for histological observation. More specific immunochemistry staining has been conducted to investigate the phenotype of the keratinocytes. Monoclonal anti-p63 was used to identify cultured keratinocytes on the gelatin-C6S-HA scaffold that keeping their phenotype. p63 is highly expressed in the basal or progenitor layers of many epithelial tissues. p63 is critical for maintaining the progenitor-cell populations that are necessary to sustain epithelial development and morphogenesis. In brief, samples were fixed with $4 \%$ formaldehyde for $2 \mathrm{~h}$. Blocking was conducted with $10 \%$ goat serum to prevent unspecific binding. Specimens were washed with phosphate buffered saline (PBS) for several times. Monoclonal antip63 CLONE 4A4 primary antibody (dilution $1: 200$ ) 
(Cat no. p3737 Sigma Co.) was incubated with the sample for $1 \mathrm{~h}$ at $37^{\circ} \mathrm{C}$. Next, antimouse IgG horseradish peroxidase secondary antibody (Sigma Co.) was then incubated with the previous samples for $30 \mathrm{~min}$ after specimens were washed with PBS. SIGMA FAST 3,3'-Diaminobenzidine Tablets (DAB buffered tablets, Urea Hydrogen Peroxide, Tris buffer, Sigma) was used for staining.

\section{RESULTS}

\section{Morphology of the gelatin-C6S-HA membrane}

Gelatin-C6S-HA membrane with adequate porous structure was successfully prepared by the double cross-linking and the consecutive lyophilized method. A two-layer structure with different pore sizes and porosity was clearly shown on the membrane during the SEM examination (Fig. 2). The top layer was a thin layer $(100 \mu \mathrm{m})($ Fig. $2 \mathrm{a})$ with interconnected pore size in the range of $20-50 \mu \mathrm{m}$ (Fig. 2b). This layer also developed a rete ridge-like structure and could act as a barrier to separate the dermal and epidermal layers. The interconnected pores play a major role in the communications between the keratinocytes and dermal fibroblasts, which help the keratinocytes to differentiate into a multilayer epidermis.

The bottom layer was a thicker layer (Fig. 2a; about $1 \mathrm{~mm}$ ) with an average pore size of $150 \mu \mathrm{m}$ (Fig. 2c). Dermal fibroblasts were retained in the pores of this layer, proliferated, secreted extracellular matrix, and later developed into dermis.

\section{Dermal fibroblasts seeding, migration, and proliferation}

The seeding of the dermal fibroblasts by selfdesigned spinner flask in the thicker layer (larger pores) was quite homogeneous (Fig. 3a,b). In this study, over $95 \%$ of total cells could be caught by the membrane. Dermal-fibroblast viability was investigated by MTT assay on days 1, 3, 5, and 7. Data indicated that cell numbers increased with days in three groups (on the Petri dish, gelatin scaffold, and gelatin-C6S-HA scaffold), especially in the group of gelatin-C6S-HA scaffold (Fig. 3c). The results of the total DNA analysis of gelatin, gelatin-C6S, gelatinC6S-HA are also similar (Fig. 3d). These results suggest that $\mathrm{C} 6 \mathrm{~S}$ and HA could help cell proliferation and migration into $3 \mathrm{D}$ scaffolds more effectively. At $4 \mathrm{~h}$ after seeding, dermal fibroblasts started to stick and spread out with filopodia formation (Fig. 4a). The cells also stretched out and anchored on the margins of pores during cell migration (Fig. 4b).

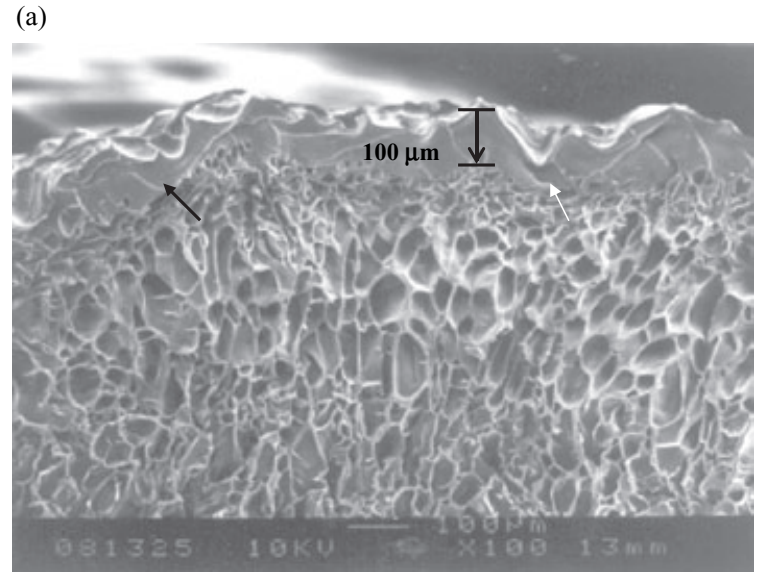

(b)

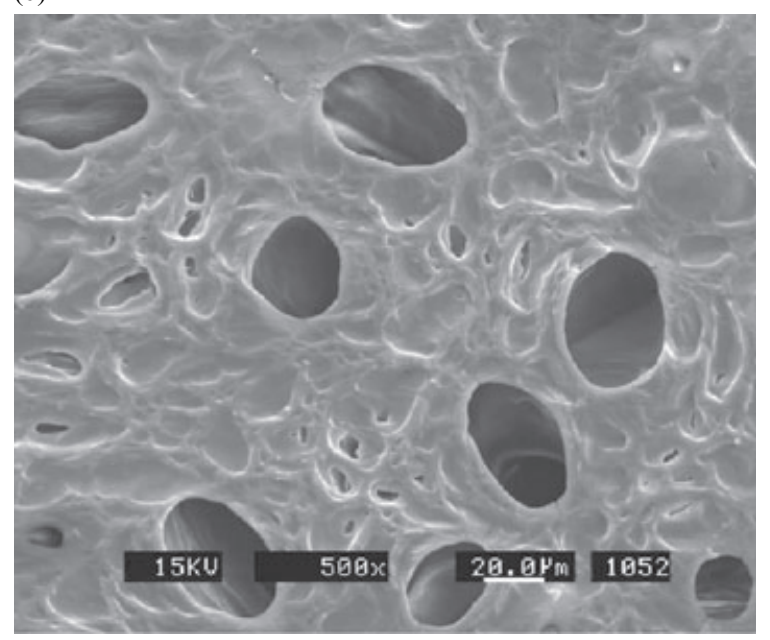

(c)

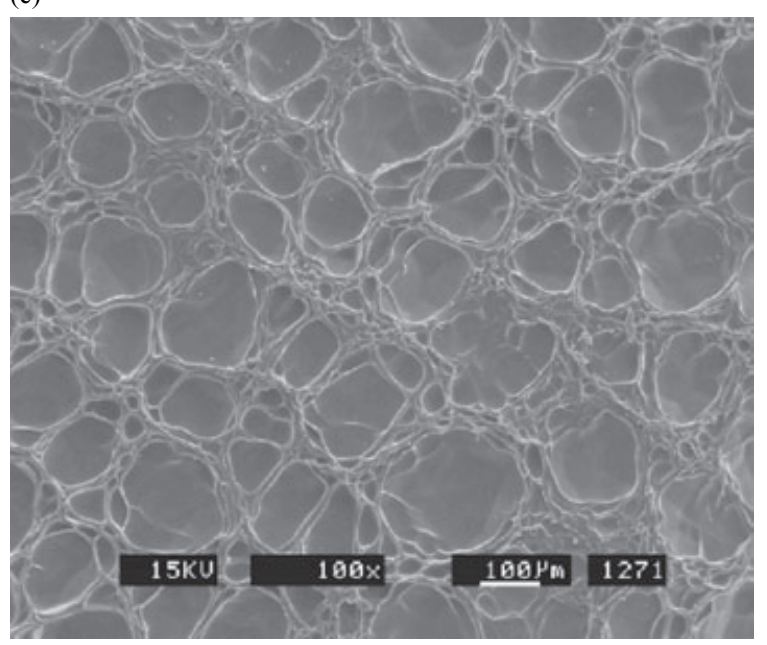

FIG. 2. Scanning electron microscope view of gelatin-C6S-HA membrane. (a) The gelatin-C6S-HA membrane showed a twolayer structure with different pore sizes and porosity in each layer. The top layer is a thinner layer with $100 \mu \mathrm{m}$ in thickness. The layer also developed like a rete ridge structure in dermis papilla (black arrow indicated). (b) The upper part (smaller pore size) of the membrane for epidermis equivalent; pore size in a range of 20-50 $\mu \mathrm{m}$. (c) The bottom layer (larger pore size) is a thicker layer with an average pore size of $150 \mu \mathrm{m}$. 
(a)

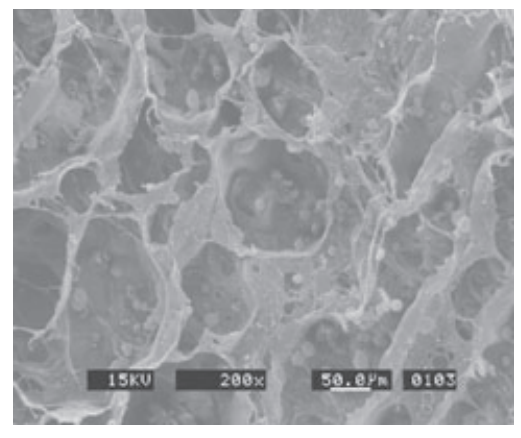

(b)

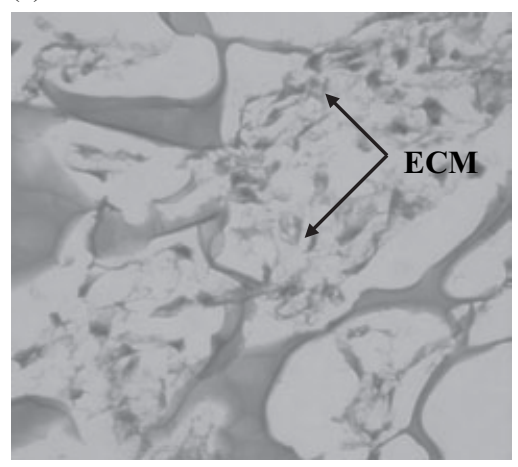

(c)

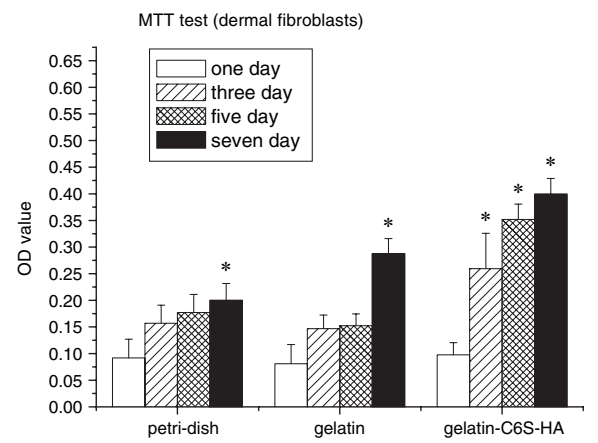

(d)

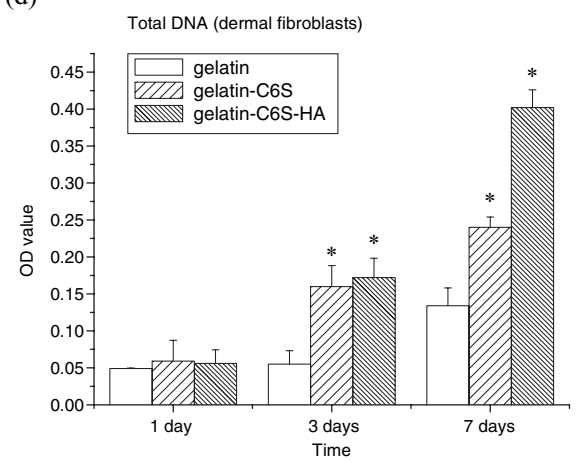

FIG. 3. The seeding, migration, and proliferation of dermal fibroblasts. (a) Dermal fibroblasts were seeded into the thicker layer (larger pores) by the self-designed spinner flask. 24 hours after seeding, the dermal fibroblasts showed a homogeneous distribution in the gelatin-C6S-HA scaffold. (b) H/E stain of dermal fibroblasts in gelatin-C6S-HA membrane (400x) (ECM, extra cellular matrix). (c, d) MTT test and total DNA analysis of dermal fibroblasts viability and growth on the petri dish, gelatin scaffold, and gelatin-C6S-HA scaffold. Data indicated that cell numbers increased with days in three groups, especially in the group of gelatin-C6S-HA. This might suggest that C6S and HA may help cells proliferate and migrate into 3-D scaffold. (One-way analysis of variance, $n=12$, Mean $\pm \mathrm{SD},{ }^{\star} P<0.05$.)

\section{Proliferation of keratinocytes}

The keratinocytes were seeded onto the upper layer (smaller pores) of the membrane 3 days after dermal fibroblasts were seeded in the larger-pores layer (Fig. 5a), and then submerged in the medium for another 5 days. After seeding, the keratinocytes progressively expanded in cell population, formed a monolayer to cover the top surface of the membrane, and secreted extracellular matrix as shown in Fig. 5b. At day 5, the monolayer organized into a keratinocyte sheet and basal lamina formed as shown in Fig. 5c.

\section{Skin equivalent formation}

After culturing the keratinocytes in a submerged condition for 5 days, the membrane was then shifted to a trans-well to remain in air-liquid interface conditions for further differentiation. The keratinocytes started to differentiate into a mutilayer structure when cultured in air-liquid interface condition for 7 days (Fig. 6a). Fourteen days later, the keratinocytes developed into an epidermis-like structure with the formation of basal layers, suprabasal layers, and the cornified squamous layer (Fig. 6b,c). Keratinocytes in the basal layer of basal lamina and basement membrane zone also maintained their phenotype (Fig. 6c). The bottom part of the membrane with dermal fibroblasts retained developed into a dermis-like structure with sparse distribution of the fibroblasts surrounding with its own extracellular matrix (Fig. 6b). This fact showed that normal keratinocyte maturation process was ongoing and the skin equivalent was formed within 21 days.

\section{DISCUSSION}

Cell adhesion is generally mediated by certain extracellular matrix proteins such as fibronectin, vitronectin, and laminin as well as various glycosaminoglycans $(17,18)$. All these adhesion-related molecules possess special amino acid sequence as binding site for cell attachment. All kinds of collagen also have special sequences for cell adhesion. Gelatin is a denatured collagen without 3-D structure. However, it does have amino acid sequences such as arginine-glycine-aspartic acid (RGD) and aspartic acid-glycine-glutamic acid-arginine (DGER) sequence to help cell attachment (19). In addition, gelatin is less expensive and has lower antigenicity than that of collagen. The degraded product of gelatin is nontoxic and can provide the nutrients for skin regeneration. In the study, gelatin was used as the major matrix for scaffold design to help cell attachment and nutrient supply.

To mimic the composition of skin, C6S and HA were incorporated into a gelatin scaffold to provide a better environment for cell proliferation and 
(a)

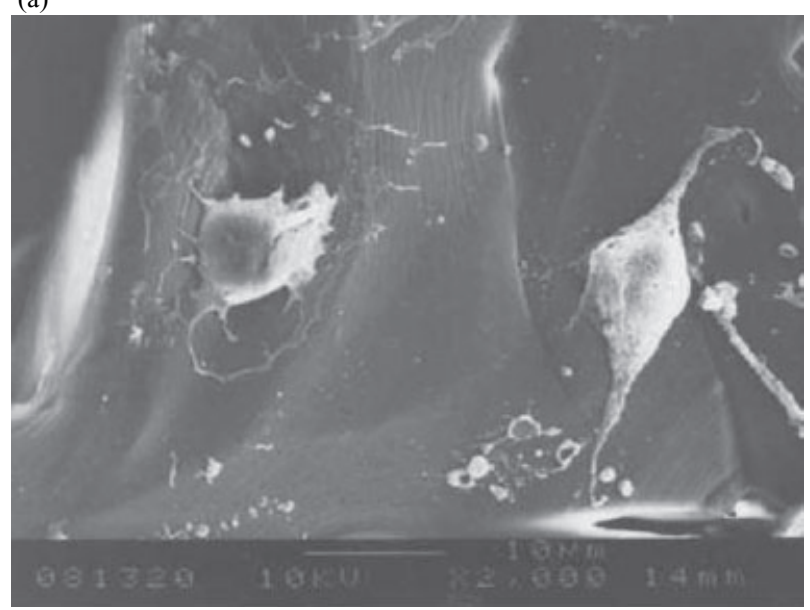

(b)

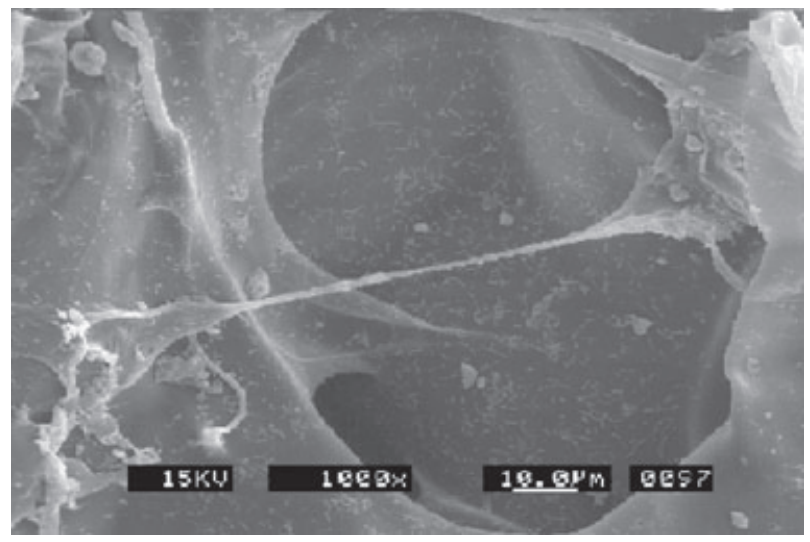

FIG. 4. Scanning electron microscopic examination of the morphologic changes of dermal fibroblasts. (a) After being seeded, dermal fibroblasts started to stick and spread out the filopodia during the initial $4 \mathrm{~h}$. (b) The cells also stretched out the anchoring structures during cell migration from one place to another.

migration (Fig. 3c,d). HA, with its repeating disaccharide sequence, is endogenous to normal skin and has been implicated in a scarless fetal healing process $(20,21)$. It was reported to promote cell migration (22) and resulted in better cell distribution in the porous scaffold of this study (Fig. 3a,b). It was reported that fibroblasts carry receptors, such as CD44, allowing specific attachment to and migration along HA molecules. Furthermore, CD44 ligands like HA and C6S may inhibit macrophage multinucleation followed by reduced inflammatory response (23). The polysaccharides possess high water-binding capacity, which will be helpful for retaining large porous lamellar matrix spaces, promoting matrix swelling and absorbing wound exudates during clinical application (Fig. 2). In the normal skin structure, the C6S exists mainly in the layer of basal lamina (24). (a)

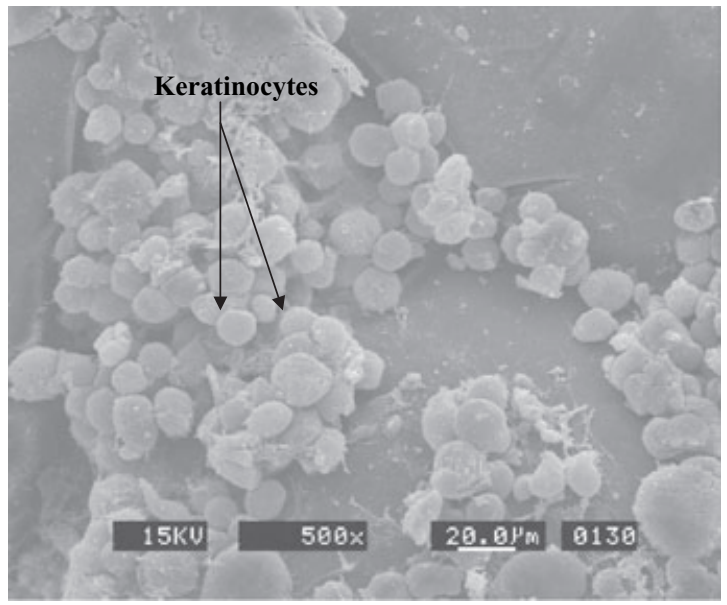

(b)

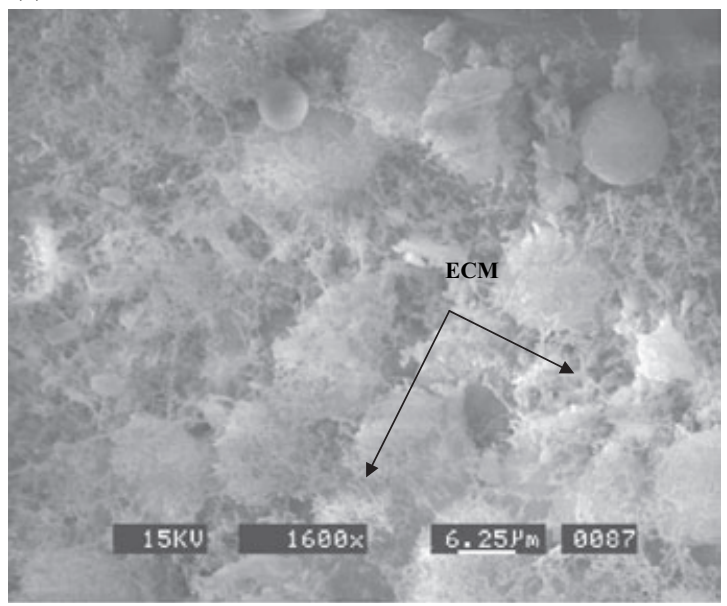

(c)

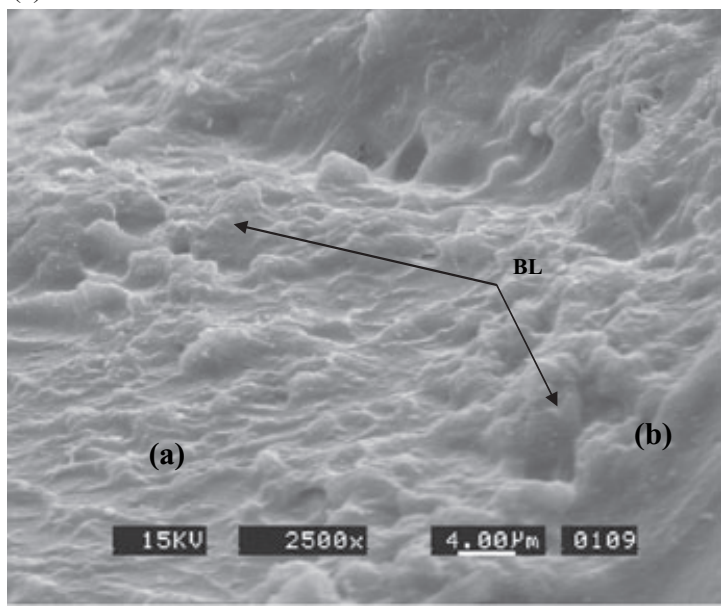

FIG. 5. Scanning electron microscopic examination of the morphologic changes of keratinocytes. (a) Keratinocytes were initially seeded onto the upper layer (smaller pores) of the gelatin-C6SHA membrane. (b) The keratinocytes progressively expanded in number and formed a monolayer to cover the top surface of the scaffold and started to build up basement membrane. (arrow indicated). (c) At day 5, the monolayer organized into a keratinocytes sheet and basal lamina. (ECM, extracellular matrix; BL, basal lamina.) 
During the works of tissue engineering, a bioreactor should offer an environment to seed cells (in this study, the dermal fibroblast seeding) in scaffold homogeneously (25). For the self-designed bioreactor used in this study, the membranes were put into both ends of the tissue cassette with a distance of $0.5 \mathrm{~cm}$ away from the central axis (Fig. 1). A $2.5-\mathrm{cm}$ magnetic stir bar was spun at a rate of $80 \mathrm{rpm}$. The dermal fibroblasts were suspended and circulated on a constant track due to the balance of cell density and spin rate, just like satellites around a planet. Thus, the dermal fibroblasts could be caught at a certain distance away from the central axis and on one side of the membrane. The gelatin helps the cells attach. HA promotes cellular migration. The negative charge of $\mathrm{C} 6 \mathrm{H}$ and $\mathrm{HA}$ provides greater water absorption ability, and creates large porous lamellar matrix spaces. The self-designed bioreactor catches cells on a constant track. All these factors contribute to an optimum scaffold for cell adhesion, migration, and distribution.

In the process of harvesting cultured epithelial sheet grafts, the basement membrane proteins of the harvested graft may be inevitably digested by the digesting enzyme, thus affecting its anchorage on the recipient wound bed (26). Shrinkage of the harvested grafted sheet and blister formation below the graft are two major drawbacks of conventional cultured epidermal autografts (27). To avoid this potential negative aspect of enzyme treatment, we investigated the cultivation of keratinocytes directly on the gelatin-C6S-HA biopolymer membrane. We also took the advantage of inoculating dermal fibroblasts instead of using irradiated or mitomycin C-treated 3T3 cells as feeder layer. This procedure not only shortened the in vitro cultivation time but also limited the risk of disease transmission in the future. As noted in this study, the dermal fibroblast seeded layer (larger pore of the membrane) could be developed into dermal analog (Fig. 3b). The layer could act as the feeder layer of keratinocytes (on the top layer of the membrane) and allows them to proliferate without losing their phenotype (Fig. 6c).

The diffusion of nutrition supply from the recipient wound is critical especially in the first few days when neovascularization in the neodermis has not yet developed (28). The micropores invagination topography between the dermal-epidermal layers of our skin equivalent showed the possibility of dynamic interactions in tissue homeostasis and served as papillary-like structures, just like rete ridges in the normal dermal-epidermal junction (Fig. 6). Researchers have elucidated that the dermal fibroblasts can interact with the keratinocytes (a)

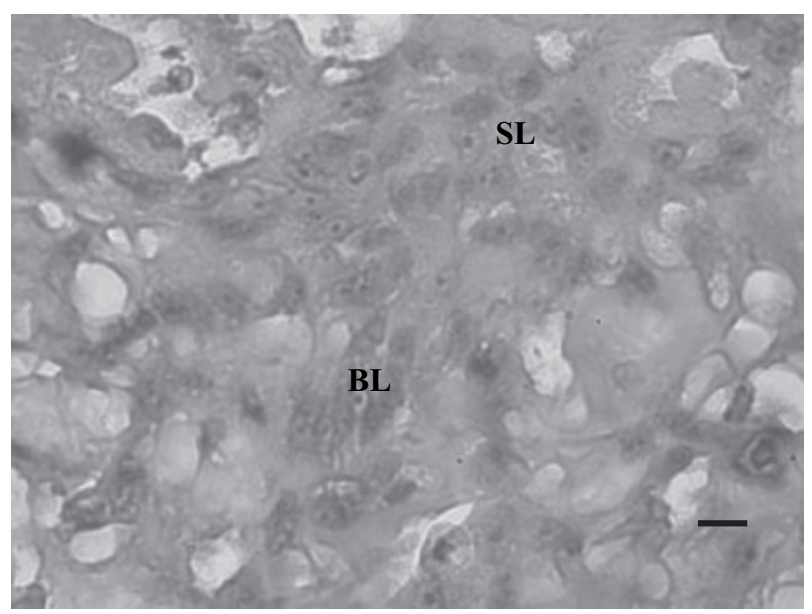

(b)

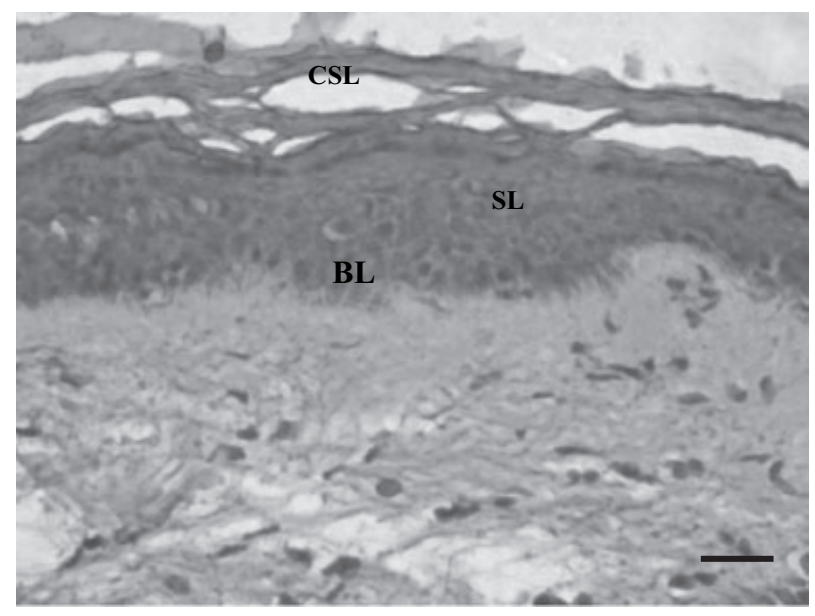

(c)

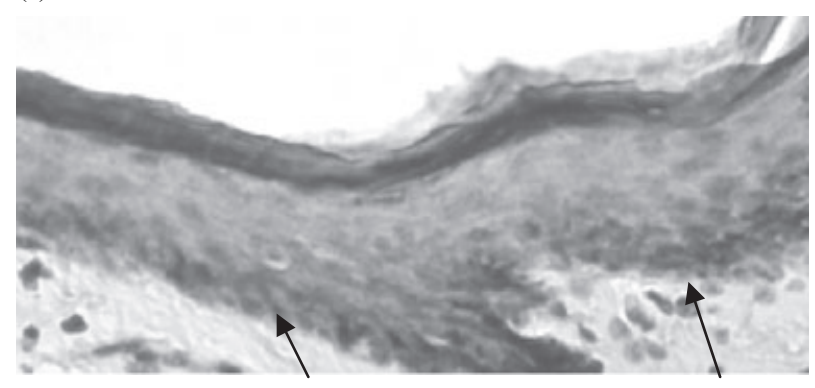

FIG. 6. Histology of culture of the keratinocytes seeded membrane in the air-liquid interface. (a) When cultured for 7 days, the keratinocytes started to differentiate into a mutilayer structure $(400 x)$. (b) After culture for 21 days, the top layer of the membrane with keratinocytes seeded developed into an epidermis-like structure with suprabasal layers and the cornified squamous layer (40x). The bottom part of the membrane with dermal fibroblasts retained developed into dermis structure with sparse distribution of the fibroblasts surrounded with their own extracellular matrix. (SL, suprabasal layer; CSL, cornified squamous layer; BL, basal lamina). (c) Monoclonal anti-p63 CLONE 4A4 was used to evaluate the kertinocytes in the basal layer of basal lamina and basement membrane zone. There was dark brown pigmentation in the bottom of the culture area (arrow) (40x). ([a] Bar $10 \mu \mathrm{m}$, [b][c] Bar $100 \mu \mathrm{m}$.) 
through cytokine release, which would promote keratinocyte differentiation to organize into an epidermis (29). The pore size was also important as a determinant of cell migration rate inside the membrane as well as the time constant of biodegradation (30). In this study, the micropores $(20-50 \mu \mathrm{m})$ not only allow the migration of the keratinocyte populations from the upper surface to the underneath recipient wound bed, but also serve as a substrate to prevent the keratinocytes from overspreading into the dermal layer. The dermal fibroblasts seeded on the macropore $(150 \mu \mathrm{m})$ part of the membrane could act as feeder layer for keratinocytes, and the porous structure of the membrane can be used for the drainage of wound exudates. Moreover, the interconnected porous structure in the scaffold would provide a chance for cytokines and growth factors to modulate epidermalization (3133). We have conducted the related experiments in our next study.

\section{CONCLUSION}

In this study, a bilayered gelatin-C6S-HA membrane with different pore sizes on both sides has been developed. The lower layer of the membrane with pore sizes of $150 \mu \mathrm{m}$ would be seeded with dermal fibroblasts for dermis development and also act as the feeder layer for keratinocytes inoculation. The upper layer with pore size about $20-50 \mu \mathrm{m}$ was seeded with keratinocytes for epidermalization. The keratinocytes were cultured in submerged conditions for 5 days and then kept in air-liquid interface conditions for further differentiation and maturation. After being cultured in air-liquid interface conditions for 21 days, the kertinocytes differentiated into an epidermis-like structure with suprabasal layers and the cornified squamous layer of normal skin. The bottom part of the membrane with dermal fibroblasts retained was developed into a dermis structure with sparse distribution of the fibroblasts surrounded with its own extracellular matrix. This newly designed skin equivalent was well-formed within 21 days, comparable to other cultured skin substitutes. This tissue-engineered skin substitute will be used as an investigation model for in vitro toxicology, cutaneous physiology examination, and in vivo wound healing process in the near future.

Acknowledgments: The authors would like to express their appreciation to the National Science Council of ROC for their financial support to the research.

\section{REFERENCES}

1. Peeters R, De Caluwe D, Neetens C. Use of glycerolized cadaver skin for the treatment of scalds in children. Burns 1994;20:32-3.

2. Spence RJ, Wong L. The enhancement of wound healing with human skin allograft. Surg Clin North Am 1997;77:731-45.

3. Greeleaf G, Hansbrough JF. Current trends in the use of allograft skin for patients with burns and reflections on the future of skin banking in the United States. J Burn Care Rehabil 1994;15:428-31.

4. Kremer M, Lang E, Berger AC. Evaluation of dermalepidermal skin equivalents ("composite-skin") of human keratinocytes in a collagen-glycosaminoglycan matrix (Integra Artificial Skin). Brit J Plast Surg 2000;53:459-65.

5. Medalie DA, Eming DA, TompKins RG, Yarmush ML, Krueger GG, Morgan JR. Evaluation of human skin reconstituted from composite grafts of cultured keratinocytes and human acellular dermis transplanted to athymic mice. J Invest Dermatol 1996;107:121-7.

6. Rheinwald JG, Green H. Serial cultivation of strains of human epidermal keratinocytes: the formation of keratinizing colonies from single cells. Cell 1975;6:331-44.

7. Rheinwald JG, Green H. Epidermal growth factor and the multiplication of cultured human epidermal keratinocytes. Nature 1977;265:421-4.

8. Green H, Kehinde O, Thomas J. Growth of cultured human epidermal cells into multiple epithelia suitable for grafting. Proc Natl Acad Sci USA 1979;76:5665-8.

9. Woodley DT, Peterson HD, Herzog SR, et al. Burn wounds resurfaced by cultured epidermal autografts show abnormal reconstitution of anchoring fibrils. JAMA 1988;259:2566-71.

10. Butler CE, Yannas IV, Compton CC, Correia CA, Orgill DP. Comparison of cultured and uncultured keratinocytes seeded into a collagen-GAG matrix for skin replacements. Brit J Plast Surg 1999;52:127-32.

11. Ramos-e-Silva M, Ribeiro de Castro MC. New dressings, including tissue-engineered living skin. Clin Dermatol 2002; 20:715-23.

12. Kearney JN. Clinical evaluation of skin substitutes. Burns 2001;27:545-51.

13. Pruitt BA Jr, Levine NS. Characteristics and uses of biologic dressings and skin substitutes. Arch Surg-Chicago 1984;119: 312-22.

14. Kemp P, Bell E, Kagan DT, Mason V, Cavallaro J. Preparation of tissue equivalents by contraction of a collagen gel layered on a collagen gel. US Patent 5,536,656, 1996.

15. Michel M, L'Heureux N, Pouliot R, Xu W, Auger FA, German L. Characterization of a new tissue-engineered human skin equivalent with hair. In Vitro Cell Dev-An 1999;35:318-26.

16. Mosmann T. Rapid colorimetric assay for cellular growth and survival: application to proliferation and cytotoxicity assay. J Immunol Methods 1983;65:55-63.

17. Culp LA, Murray BA, Rollins BJ. Fibronectin and proteoglycan as determinants of cell-substratum adhesion. J Supramol Str Cell 1979;11:401-27.

18. Hersel U, Dahmen C, Kessler H. RGD modified polymers: biomaterials for stimulated cell adhesion and beyond. Biomaterials 2003;24: 4385-415.

19. Davis DH, Giannoulis CS, Johnson RW, Desai TA. Immobilization of RGD to $<111>$ silicon surfaces for enhanced cell adhesion and proliferation. Biomaterials 2002;23:4019-27.

20. Krummel TM, Mast BA, Haynes JH, Diegelmann RF, Cohen IK. Characteristics of fetal repair. Prog Clin Biol Res 1991; 365:167-76.

21. Longaker MT, Adzick NS. The biology and therapeutic applications of fetal wound healing. Clin Mater 1991;8:223-7.

22. Hall CL, Wang C, Lange LA, Turley EA. Hyaluronan and the hyaluronan receptor RHAMM promote focal adhesion turnover and transient tyrosine kinase activity. J Cell Biol 1994;126:575-88. 
23. Sterling H, Saginario C, Vignery A. CD44 occupancy prevents macrophage multinucleation. J Cell Biol 1998;143:837-47.

24. Chan L, Wong YC. Cytochemical localization and characterization of proteoglycans (glycosaminoglycans) in the epithelial-stromal interface of the seminal vesicle of the guinea pig. J Anat 1992;180:41-56.

25. Wang HJ, Bertrand-de Hass M, van Blitterswijk CA, Lamme EN. Engineering of a dermal equivalent: seeding and culturing fibroblasts in PEGT/PBT copolymer scaffolds. Tissue Eng 2003;9:909-17.

26. Germain L, Rouabhia M, Guignard R, Carrier L, Bouvard V, Auger FA. Improvement of human keratinocyte isolation and culture using thermolysin. Burns 1993;19:99-104.

27. Boyce ST, Supp AP, Harriger MD, Greenhalgh DG, Warden GD. Topical nutrients promote engraftment and inhibit wound contraction of cultured skin substitutes in athymic mice. J Invest Dermatol 1995;104:345-9.

28. Tsuzuki Y, Fukumura D, Oosthuyse B, Koike C, Carmeliet P, Jain RK. Vascular endothelial growth factor (VEGF) modulation by targeting hypoxia-inducible factor-1alpha $\rightarrow$ hypoxia response element $\rightarrow$ VEGF cascade differentially regulates vascular response and growth rate in tumors. Cancer Res 2000;60:6248-52.

29. Huang YC, Wang TW, Sun JS, Lin FH. Cultured keratinocytes and dermal fibroblasts on a double layer scaffold with bimedium culture system. Biomed Sci Instrum 2003;39:500-5.

30. Dagalakis N, Flink J, Stasikelis P, Burke JF, Yannas IV. Design of an artificial skin. Part III. Control of pore structure. J Biomed Mater Res 1980;14:511-27.

31. El Ghalbzouri A, Ponec M. Diffusible factors released by fibroblasts support epidermal morphogenesis and deposition of basement membrane components. Wound Repair Regen 2004;12:359-67.

32. Coulomb B, Lebreton C, Dubertret L. Influence of human dermal fibroblasts on epidermalization. J Invest Dermatol 1989;92:122-5.

33. Demarchez M, Hartmann DJ, Regnier M, Asselineau D. The role of fibroblasts in dermal vascularization and remodeling of reconstructed human skin after transplantation onto the nude mouse. Transplantation 1992;54:317-26. 